\title{
The Elusive Bose Metal
}

\author{
Philip Phillips,${ }^{1 *}$ Denis Dalidovich, ${ }^{2}$ \\ ${ }^{1}$ Loomis Laboratory of Physics, University of Illinois at Urbana-Champaign, \\ 1100 W.Green St., Urbana, IL., 61801-3080 \\ ${ }^{2}$ National High Field Magnetic Laboratory,Florida State University \\ Tallahassee, Florida 32310 \\ *To whom correspondence should be addressed; E-mail: dimer@uiuc.edu.
}

The conventional theory of metals is in crisis. In the last 15 years, there has been an unexpected sprouting of metallic states in low dimensional systems directly contradicting conventional wisdom. For example, bosons are thought to exist in one of two ground states: condensed in a superconductor or localized in an insulator. However, several experiments on thin metal alloy films have observed that a metallic phase disrupts the direct transition between the superconductor and the insulator. We analyze the experiments on the insulatorsuperconductor transition and argue that the intervening metallic phase is bosonic. All relevant theoretical proposals for the Bose metal are discussed, particularly the recent idea that the metallic phase is glassy. The implications for the putative vortex glass state in the copper-oxide superconductors are examined.

Before the advent of quantum mechanics, the microscopic origin of electrical conduction in metals remained shrouded in a central mystery: How do electrons avoid the atoms in a dense material? Realising that electrons move as waves, Bloch (1) proposed that electrons surf the 
atoms in a crystal by adjusting their wavelength to fit the periodicity of the lattice. Bloch's view is radical in that electron-electron interactions are absent and defects are assumed to provide only a minor correction. We know now that both of these assumptions are wrong. For example, defects can destroy perfect conduction leading to electron localization (2) and electron interactions can generate new many-body states such as superconductivity and magnetism. Even in the extreme case in which single electrons (3 4) are localized by defect scattering, superconductivity still obtains. How is this possible? At low temperatures, lattice-mediated attractive interactions between electrons produce a resistanceless state in which the charge carriers are electron pairs, Cooper pairs. Pair formation, however, is not a sufficient condition for superconductivity. Superconductivity obtains when all of the Cooper pairs phase lock into a single quantum state. Such macroscopic occupation of a single quantum state is not possible for fermions as a result of the Pauli exclusion principle. However, Cooper pairs whose radius of gyration is smaller than the inter-pair spacing are bosons and there is no exclusion principle for bosons. Hence, macroscopic occupation of a single quantum state is permissible and it is the resultant phase coherence that thwarts localization. Superconductivity in the localized electron regime can be thought of as Bose-Einstein condensation, a phenomenon which has received much attention recently with the myriad of experiments reporting Bose superfluidity in optical lattices of alkali atoms (5, 6). In the other extreme where the inter-pair spacing is smaller than the Cooper pair radius, the Cooper pair $\rightarrow$ boson mapping breaks down. Nonetheless, phase coherence still obtains. Consequently, we will adopt the simplification in this article that Cooper pairs are simply charge $2 e$ bosons, $e$ the electron charge.

Because bosons can superconduct, one might presuppose that they can also exist in a metallic state. However, as we will see, a simple quantum mechanical principle precludes this state of affairs. In low dimensions ( $D \leq 2)$, except in the presence of correlated disorder $(7)$ or a magnetic field $(8 \mid 9)$, not even non-interacting electrons can remain metallic at zero temperature in 
the presence of disorder. When the interactions are weak and repulsive, the ground state appears to be ferromagnetic (10, 11, 12) rather than metallic (13). However, recent experiments (14) on 2D semiconductor heterostructures indicates that in the strongly interacting regime, a metallic state forms. At present, this problem is unresolved (14). The construction of bosonic or electronic metallic states in 2D remains one of the grand challenges in solid state physics. We are concerned in this review with the possible existence of a metallic state for bosons in $2 \mathrm{D}$.

\section{Phase and Particle Number Duality}

To understand why a Bose metal poses a serious theoretical question, we review the two standard ways in which superconductivity can be destroyed. Pair formation and phase coherence underlie superconducting order. Consequently, we represent the order parameter, $\Psi(\mathbf{r})=$ $\Delta \exp (i \phi(\mathbf{r}))$, for a superconductor as a simple product of the pair amplitude, $\Delta$ and a rigid time-independent phase, $\phi(\mathbf{r})$. In terms of the original electron coordinates,

$$
\Psi(\mathbf{r})=\left\langle c_{\downarrow}(\mathbf{r}) c_{\uparrow}(\mathbf{r})\right\rangle=\langle\psi(\mathbf{r})\rangle
$$

where $c_{\sigma}(\mathbf{r})$ annihilates an electron with spin $\sigma$ at $\mathbf{r}$, the angle brackets indicate an average over the quantum states of the system and $\psi(\mathbf{r})$ is the wavefunction for a Cooper pair. Phase coherence occurs when the phase correlator $G(\mathbf{r})=\langle\psi(\mathbf{r}) \psi(0)\rangle$ approaches a non-zero value

for large $\mathbf{r}$. However, this rule is too restrictive. In 2D, $G(\mathbf{r}) \propto r^{-\eta}$ decays algebraically (15 16) at the finite temperature superconducting transition, with $0<\eta<1$. Herein lies a key difference between superconductivity in 2D and bulk systems. Because of the algebraic fall-off of phase coherence in 2D, the temperatures at which the Cooper pair amplitude first becomes non-zero and global phase coherence occurs can differ substantially. We will refer to the Cooper pair onset temperature as $T_{c 0}$ and the phase ordering temperature as $T_{c}$.

Consequently, superconductivity is destroyed by either breaking the Cooper pairs or disrupting phase coherence. As a result of the localization principle in $2 \mathrm{D}$, an insulating state nec- 
essarily results in either case: single electrons are localized by disorder and phase-incoherent Cooper pairs are limited spatially by the localization length. However, an even more profound quantum principle underlies localization in the latter case. In the localized boson regime, snap shots at varying times of regions of the sample on the scale of the localization length reveal no fluctuation in the number of Cooper pairs. However, in the superconducting state, analogous snap shots reveal that the Cooper pair number varies wildly (Fig. 1). The simple reason is that phase and particle number are conjugate variables and hence, their simultaneous measurement is limited by the Heisenberg uncertainty principle. Consequently, bosons can either be in an eigenstate of particle number or phase. The eigenstate of phase is a superconductor and that of particle number is a localized insulator. No other possibilities exist. At the transition point between the insulator and superconductor, Cooper pairs on the brink of losing phase coherence diffuse (17 [18) with a conductance whose scale is set by the quantum of conductance for charge 2e bosons, namely $(2 e)^{2} / h$ where $h$ is Planck's constant. The coefficient of this conductance is not necessarily unity (19) as will be seen.

Experimentally, one transforms a superconductor into an insulator by changing the intensity of the laser light in optical lattices (6) or for thin films by either decreasing the film thickness (20, 21) or applying a perpendicular magnetic field (22] 23, 24 25] 26). Decreasing the thickness increases boundary scattering and hence disorder drives the transition, whereas in the application of a magnetic field, resistive topological excitations called vortices (the dual of Cooper pairs) frustrate the onset of global phase coherence. As long as the two ground states on either side of the transition correspond to condensed and localized bosons, the transition is controlled by quantum mechanics in the sense that a $T=0$ quantum critical point governs the physics. Consequently, the physics should be independent of the tuning parameter. Early experiments on the field-tuned insulator-superconductor transition (IST) in $\operatorname{InO}_{x}(22)$ films and disorder-tuning in Bi deposited on Ge (21) seemed to fit the expected paradigm shown in Fig. 
(11). Namely, below a critical value of the magnetic field or the disorder, a superconducting state obtained with a vanishing resistance and beyond, the resistivity turned upwards as the temperature was decreased indicating an insulating state, in apparent with theory.

\section{Experiment: Bose Metal}

However, such agreement did not last long. As early as 1989, Goldman and collaborators (20) observed that in a wide array of homogeneously disordered films ranging from $\mathrm{Ga}, \mathrm{Al}$, $\mathrm{Pb}$, to In, continuously decreasing the thickness led first to a leveling of the resistance at low temperatures immediately after superconductivity was destroyed. Homogeneously disordered films, that is films in which the disorder is on atomic scales, can be produced by sputtering or from films deposited on specially prepared substrates cooled to liquid helium temperatures (20). The data shown in Fig. (2) taken on Ga deposited on Alumina demonstrate that the leveling extrapolates smoothly to $T=0$, indicating the emergence of a true metallic phase. Unlike the earlier films which were homogeneous, the Ga/alumina films were granular. Let $g$ be the coupling constant (in this case the amount of disorder) that drives the destruction of superconductivity and $g_{c}$ the critical value at the onset of the metallic state. Fig. (2) suggests that the $T=0$ value of the resistivity in the metallic phase is critical in that it scales as some power of $\left(g-g_{c}\right)$, thereby vanishing at criticality. This observation will play a central role in constructing a theory for the metallic phase. For sufficiently thin films, insulating behavior obtains. What about field-tuning? In a magnetic field, vortices of only one vorticity are possible in the superconducting phase. At low temperatures and non-zero magnetic fields, an exponentially small resistivity of the form $R(T) \propto \exp -C|\ln T| / T$ is expected on the superconducting side as a result of thermally assisted quantum tunneling of vortices. However, several groups (23| 24 29) have observed a leveling (see Fig. (3)) of the resistance here as well rather than the expected exponential dependence. In fact, similar behavior has been observed in graphite (27). That the metallic state is really an artifact of failed refrigeration has been addressed by minimizing 
power dissipation (20) and by the observation that in the field-tuned samples (24), the effective temperature associated with the high temperature activated behavior varied systematically with magnetic field. Further, the deviation (24) from activated behavior occurred at a lower temperature as the field increased, where power dissipation and sample heating effects are greatest. Hence, the metallic state is not a simple artifact of heating. More recent experiments (25) on the low-field regime reveal that true superconductivity with a vanishing resistivity persists only until a magnetic field of magnitude $H_{M}=0.018 T$. The turn-on of the resistivity at $H_{M}=0.018 T$ is shown clearly in Fig. 4 Insulating behavior does not obtain until $H>H_{c}=1.8 T$. Consequently, the metallic phase persists over a wide range of magnetic field, $0.018<H<1.8 T$. Also apparent from Fig. 4 is that the resistivity at a fixed temperature and magnetic field is not unique: cycling the magnetic field up and then down changes the resistivity, an indication that the energy landscape has numerous metastable states as in the case of window glass, for example.

\section{Electron Insulator}

Of course, the observation of a metallic phase poses an additional problem: What is the nature of the insulating phase? This problem surfaces because if a metallic state exists, then the insulator cannot arise simply by breaking phase coherence. Perhaps the pairs fall apart to form the insulator? This is precisely what was (30) concluded from tunneling measurements which yield a linear relationship with unit slope between the pair amplitude, $\Delta$ and $H_{c}$. This relationship holds only if $H_{c}$ corresponds to the field at which Cooper pairs disintegrate. It was also noticed (23) that the value of $H_{c}$ was identical to the known value of the magnetic field at which Cooper pairs fall apart in MoGe. Hence, it is reasonable to conclude (31 32) that it is electronic excitations rather than localized Cooper pairs that ultimately drive the insulating state at least in the homogeneously disordered films. On this reading, it is the metal that corresponds to the breaking of phase coherence and as a result, bosons carry the current. 


\section{Proposed Theories}

Hence, the problem at hand is the construction of a theory for a Bose metal in 2D. Such a metal must be stable to disorder and have a resistivity that scales (see Fig. 2) as some power of the distance from criticality, $g-g_{c}$. Because the basic excitations are Cooper pairs, models with superconducting grains have been used widely to solve this problem (34 33, 36 35). In such models, Cooper pairs tunnel between the grains with an amplitude, $J$, and the charging energy of each grain is $E_{C}$. Some have put aside the issue of the origin of the metallic state (33, 34) and considered instead an idealized model of superconducting grains embedded in a metal. While the superconducting side is complicated by electron-hole pair scattering from normal electrons (33 34), quenching of superconductivity simply unmasks the background conductance of the host metal. Hence, by design, such models admit a direct transition from a superconductor to a metal. In the absence of the host metal, such hybrid models with normal electrons providing dissipation in an array of superconducting grains exhibit metallic behavior only at the separatrix between the insulator and the superconductor (35). In other models (36) Coulomb interactions between the superconducting grains were included. In this vein, it has been proposed (36) that beyond a critical grain size, a metallic phase obtains. This model raises the possibility that short-range Coulomb interactions lead to new physics near the quantum critical point associated with the destruction of phase coherence in an array of superconducting grains. Scaling arguments (37) indicate, however, that short-range interactions are irrelevant near the corresponding quantum critical point as long as the phases are translationally and rotationally invariant as would be the case for a Bose metal. Additionally, the Bose superfluid has been shown to form either a toroidal magnet or a Fermi liquid in the presence of strong Coulomb repulsions, neither phase of which is metallic in 2D (38). The yet more exotic model of bosons moving on a square lattice with ring exchange, that is, cyclical permutations of bosons on four mutually neighboring lattice sites has been considered (39). While the resultant phases (39) that 
are generated share much in common with metals, such as gapless excitations, a finite compressibility, and the Bose analog of a Fermi surface, the conductivity vanishes as $T^{\gamma}$ where $\gamma>0$ and hence metallic conductivity does not survive at $T=0$. Alternatively, we have pointed out (40) that the localized 'insulator' actually has a finite conductivity at zero temperature as a result of a subtle cancellation between the exponentially small density of states and the exponentially long mean-free path for bosonic quasiparticles. This result is irrelevant to the experiments because any amount of disorder reinstates the insulator.

\section{Bose Metal: Phase Glass}

So how can the experiments be explained? Some have advocated that dissipation might be the key (41 42). However, it is difficult to pinpoint an external source of dissipation that survives at zero temperature. Further, recent work (43.44) suggests that the standard treatment of dissipation in models for phase fluctuations in an ordered array of superconducting grains cannot produce a metallic phase at $T=0$ even in the presence of electronic excitations (35). Consequently, it is advisable to focus on new phases in which dissipation is self-generated. As an example, consider the quantum phase glass model recently proposed (45) in which disorder in the distribution of tunneling amplitudes, $J_{i j}$ and quantum fluctuations destroy phase coherence. To describe superconductivity and to capture the frustration required for glassy ordering, the distribution of $J_{i j}$ 's must have a non-zero mean and contain both $\pm J$, respectively (43). While it might seem strange that the the sign of $J$ is random, this state of affairs is inextricable from the role disorder plays in a superconductor as a direct consequence (46) of exchange effects arising from the transport of a Cooper pair through a localized defect. Hence, models with purely on-site disorder are inadequate. While global phase coherence is absent from the glassy phase, locally the phase on each lattice site points along a fixed direction. However, the directions differ from site to site as illustrated in Fig. 5] Consequently, in the phase or rotor glass, the phases on each site are frozen along mutually non-collinear directions such that 
$\left\langle\exp \left(i \phi\left(r_{j}\right)\right)\right\rangle \neq 0$ but $\sum_{j}\left\langle\exp \left(i \phi\left(r_{j}\right)\right)\right\rangle=0$. Here, the angle brackets indicate an average over the distinct quantum states of the glass. The effective order parameter (47) for the glassy phase, $Q(\tau)=\left\langle\exp \left(i \phi\left(r_{j}, 0\right)\right) \exp \left(i \phi\left(r_{j}, \tau\right)\right\rangle \neq 0\right.$, reflects the local breaking of spin rotation invariance. Classically, $Q(\tau)$ relaxes exponentially fast to its equilibrium value. However, quantum mechanically (48 49), $Q(\tau)$ decays as $\tau^{-2}$. Such sluggish phase relaxation gives rise to a density of low-lying excitations that scales as $|\omega|$ and hence exceeds the standard $\omega^{2}$ dependence of the density of states in a superconductor. Such low-lying excitations have two key profound effects. First, the effective dimension of the phase glass is now $d_{\mathrm{eff}}=2+2=4$, the additional dimensions arising from the time dynamics. Second, in the total free energy in the glassy phase, such excitations couple directly to the bosonic excitations from the fluctuations of the superconducting order parameter. Bosons moving in such a glassy environment with a myriad of metastable states fail to localize (45) because no true ground state exists. The result (45) is a metal at $T=0$ with a critical resistivity that scales as $\left(g-g_{c}\right)^{p}$, where $p>0$, consistent with experiment (see Figs. 2 and 4 ). This result holds even when the bosons are allowed to interact (45). Further, the vanishing of the resistivity at the critical point is consistent with a recent analysis (19) that demonstrates that at criticality, the resistivity does depend universally on $h / 4 e^{2}$. However, the prefactor is zero (19). Hence, the phase glass is a candidate to explain the intervening metallic phase.

\section{Is a Phase Glass Stiff?}

Is a phase glass a superconductor in disguise? If yes, then the metallic behavior found above, while intriguing, will be dwarfed by the infinite contribution to the conductivity arising from the collective mode (or phase stiffness) of the phase glass. This question is relevant to topological glasses in general, in particular the vortex glass (50) which has been argued to have zero resistance and to explain the ground state of copper oxide superconductors in a perpendicular magnetic field. The vortex glass state is currently controversial because extensive 
experiments on copper oxide samples designed to optimize the conditions for the validity of the vortex glass model, namely untwinned (that is no grain boundaries and hence the defects are point-like) $\mathrm{YBa}_{2} \mathrm{Cu}_{3} \mathrm{O}_{7-\delta}(\mathrm{YBCO})$ crystals, find an absence of scaling and a non-vanishing linear resistivity below the putative glass transition (51]52). While vortex and phase glasses are not identical, the conclusions regarding the stiffness should be model independent simply because global rotation of all the phases (or spins) around any particular axis (a generator of the group $\mathrm{SO}(\mathrm{m})$ ) leaves the free energy invariant. However, the rotated and unrotated states are distinct. As a consequence, glasses of this type break $\mathrm{SO}(\mathrm{m})$ symmetry and a gapless mode should exist. Technically, the phase stiffness is defined by applying a twist to all of the spins and determining the resultant change in the free energy. If what results can be written simply as a ballistic mode dispersing as a linear function of momentum, as in the dispersion of light, for example, then a phase stiffness exists. However, all exact calculations at long-times in either the Heisenberg (53) 54) or quantum rotor glass (55) find that the equilibrium stiffness vanishes. Nonetheless, there is a massless mode (53 54 45) that disperses as $\omega \propto i k^{2}$. At short times where the spin glass lives in a single valley, the stiffness (56) is non-zero. It is this single-valley view (infinitely high barriers) that undergirds the vortex glass (50). The physical reason for this difference is simple (see Ref. 44, p. 945) in that a spin glass is stiff if it is not allowed to hop (tunnel in the quantum rotor glass) from valley to valley as the spins are twisted. Hence, in a full statistical mechanical calculation in which all possible configurations are included as the twist is applied, the stiffness vanishes (53 54 55 47). So which result is relevant to experiments? In a true DC measurement, $\omega \ll \omega_{g}(T)$, where $\omega_{g}(T)$ is the barrier hopping frequency, the full statistical mechanical treatment is relevant and no stiffness should be expected. Although $\omega_{g}(T)$ is not known, the systematic fluctuations in the leveled region of the conductivity in Fig. 2 indicate that the measurements are in the true DC limit. Similarly, in the mixed state of the copper oxides, the non-vanishing of the resistivity below (51]52] 57) the putative vortex glass 
transition suggests that it is the equilibrium stiffness that is relevant.

\section{Final Remarks}

The observation of a metallic phase in thin metal alloy films requires a new state of matter that does not fall prey to the standard localization principles for electrons or bosons. In the presence of disorder, superconductors lose phase coherence and become glassy. Bosons moving in the resultant glassy background remain metallic at $T=0$ provided that the low-lying excitation spectruum scales as a linear function of frequency as in the phase glass. The corresponding result for the vortex glass is currently not known. Nonetheless, as we have seen, whether topological glasses have a phase stiffness is a matter of time scales. All such glasses (the vortex glass included) lose their single-valley stiffness in the long-time limit. Perhaps the persistence of a linear current-voltage response in YBCO below the putative vortex glass transition (51 52) is fundamentally telling us that the configuration of the vortices in the glassy state is not static and perhaps vortices freeze more like window glass as has been advocated (57). Hence, our proposal that the Bose metal is glassy resonates with the experimental results on the mixed state of the cuprates. While the hysteresis shown in Fig. 4 is symptomatic of glassiness, additional experiments such as noise, relaxation time measurements, as well as $T \rightarrow 0$ transport studies on the mixed state of the cuprates are needed to confirm the Bose metal picture. Indeed, glasses with their intrinsic low-lying excitations offer a glimpse into the route by which bosons circumvent localization in the presence of disorder.

We thank A. Goldman and D. S. Fisher for useful discussions and the NSF Division of Materials Research and the ACS Petroleum Research Fund for supporting this research and S. Sachdev for Fig. 1. 


\section{References and Notes}

1. F. Bloch, Z. Physik. 52, 555 (1928).

2. E. Abrahams, P. W. Anderson, D. C. Licciardello, T. V. Ramakrishnan, Phys. Rev. Lett. 42, 673 (1979).

3. M. Ma, P. Lee, Phys. Rev. B, 32, 5658 (1985).

4. G. Kotliar, A. Kapitulnik, Phys. Rev. B 53 (1986), 3146.

5. M. H. Anderson, et al., Science 269, 198 (1995).

6. M. Greiner, O. Mandel, T. Esslinger, T. W. Hänsch, I. Bloch,Nature , 415, 39 (2002).

7. D. H. Dunlap, H.-L. Wu, P. Phillips, Phys. Rev. Lett. 65, 88 (1990).

8. B. L. Altshuler, A. G. Aronov, D. E. Khmel'nitskii, A. I. Larkin in Quantum Theory of Solids, edited by I. M. Lifshits (MIR Publishers, Moscow) 1980.

9. E. Prange, S. M. Girvin, Eds. The Quantum Hall Effect (Springer, Berlin 1987).

10. D. Belitz, T. R. Kirkpatrick,Phys. Rev. B 5314364 (1996).

11. C. Chamon, E. Mucciolo, Phys. Rev. Lett. 85, 5607 (2000).

12. C. Nayak, X. Yang, cond-mat/0302503.

13. A. M. Finkelshtein, Zh. Eksp. Teor. Fiz. 84, 168 (1983).

14. E. Abrahams, S. V. Kravchenko, M. P. Sarachik, Rev. Mod. Phys. 73, 251 (2001).

15. V. L. Berezinskii, Zh. Eksp. Teor. Fiz. 61, 1144 (1971).

16. J. M. Kosterlitz, D. J. Thouless, Phys. C, J. 6, 1181 (1973). 
17. M. P. A. Fisher, Phys. Rev. Lett. 65, 923 (1990).

18. I. Herbut, Phys. Rev. Lett. 81, 3916 (1998).

19. C. Chamon, C. Nayak, Phys. Rev. B 66, 094506 (2002).

20. H. M. Jaeger, et al. Phys. Rev. B 40, 182 (1989).

21. Y. Liu, A. M. Goldman, Mod. Phys. Lett. 8, 277 (1994).

22. A. F. Hebard, M. A. Paalanen, Phys. Rev. Lett. 65, 927 (1990).

23. A. Yazdani, A. Kapitulnik, A. Phys. Rev. Lett. 74, 3037 (1995).

24. E. Ephron, A. Yazdani, A. Kapitulnik and M. Beaseley, Phys. Rev. Lett. 76529 (1996).

25. N. Mason, A. Kapitulnik, Phys. Rev. B 64, 60504-1 (2001).

26. L. J. Geerligs et. al., Phys. Rev. Lett. 63, 326 (1989).

27. Y. Kopelevich, et. al. Phys. Rev. Lett., 90, 156402-1 (2003).

28. J. M. Valles Jr., R. C. Dynes, and J. P. Garno, Phys. Rev. Lett. 69, 3567 (1992).

29. J. A. Chervenak, J. M. Valles, Phys. Rev. B 61, R9245 (

30. S.-Y. Hsu, J. A. Chervenak, J. M. Valles, Phys. Rev. Lett. 75, 132 (1995).

31. A. M. Finkelshtein, Zh. Eksp. Teor. Fiz. 45, 37 (1987).

32. P. Phillips, D. Dalidovich, Phil. Mag. B, 81, 847 (2001).

33. M. V. Feigelman, A. I. Larkin, Chem. Phys. 235, 107 (1988).

34. B. Spivak, A. Zyuzin, M. Hruska, Phys. Rev. B 64, 132502-1 (2001). 
35. K. Wagenblast, A. van Otterlo, G. Schön, and G. Zimanyi, Phys. Rev. Lett. 78, 1779 (1997).

36. D. Das, S. Doniach, Phys. Rev. B 601261 (1999).

37. P. Phillips, D. Dalidovich, Phys. Rev. B 65, 81101-1 (2002). See also Eq. 4.11 in J. Cardy, Scaling and Renormalization in Statistical Physics, (Cambridge University Press, Cambridge, 1996) p. 71.

38. M. V. Feigelman, V. B. Geshkenbein, L. B. Ioffe, A. I. Larkin, Phys. Rev. B 48, 16641 (1993).

39. A. Paramekanti, L. Balents, M. P. A. Fisher, Phys. Rev. B 66, 54526-1, (2002).

40. D. Dalidovich, P. Phillips, Phys. Rev. B 64, 52507-1 (2001).

41. E. Shimshoni, A. Auerbach, A. Kapitulnik, Phys. Rev. Lett. 803352 (1997).

42. A. Kapitulnik, N. Mason, S. A. Kivelson, S. Chakravarty, Phys. Rev. B 63, 125322-1 (2001).

43. D. Dalidovich, P. Phillips, Phys. Rev. Lett. 84, 737 (2000).

44. T. K. Ng, D. K. K. Lee, Phys. Rev. B 63, 144509 (2001).

45. D. Dalidovich, P. Phillips, Phys. Rev. Lett. 89, 27001-1 (2002).

46. B. I. Spivak, S. A. Kivelson, Phys. Rev. B 43, 3740 (1991).

47. K. Binder, A. P. Young, Rev. Mod. Phys. 58, 801 (1986).

48. J. Miller, D. A. Huse, Phys. Rev. Lett. 70, 3147 (1993).

49. N. Read, S. Sachdev, J. Ye, Phys. Rev. B 52, 384 (1995).

50. D. Fisher, M. P. A. Fisher, D. Huse, Phys. Rev. B 43, 130 (1991). 
51. W. Jian, et. al., J. Phys. Cond. Matt. 9, 8085 (1997).

52. D. R. Strachan, et al. Phys. Rev. Lett. 87, 67007 (2001).

53. M. V. Feigel'man, A. M. Tsvelik, Sov. Phys. JETP 50, 1222 (1979).

54. H. Sompolinsky, G. Kotliar, A. Zippelius, Phys. Rev. Lett. 52, 392 (1984).

55. P. Phillips, D. Dalidovich, cond-mat/0305373, Phys. Rev. B (in press).

56. G. Kotliar, H. Sompolinsky, A. Zippelius, Phys. Rev. B 35, 311 (1987).

57. C. Reichhardt, A. van Otterlo, G. T. Zimányi, Phys. Rev. Lett. 84, 1994 (2000). 


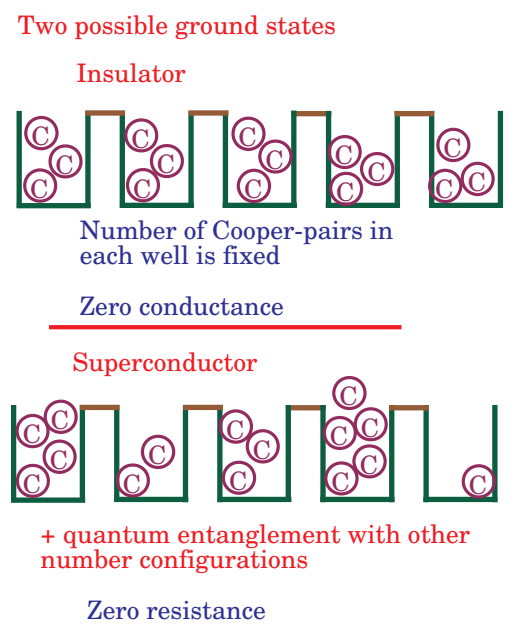

Figure 1: Insulating and superconducting ground states of Cooper (C) pairs illustrating the conjugacy between phase and number fluctuations of the Cooper pairs. In the insulator, Cooper pair number fluctuations cease leading to infinite uncertainty in the phase. Contrastly, in a superconductor, phase coherence obtains, leading thereby to infinite uncertainty in the Cooper pair particle number. 


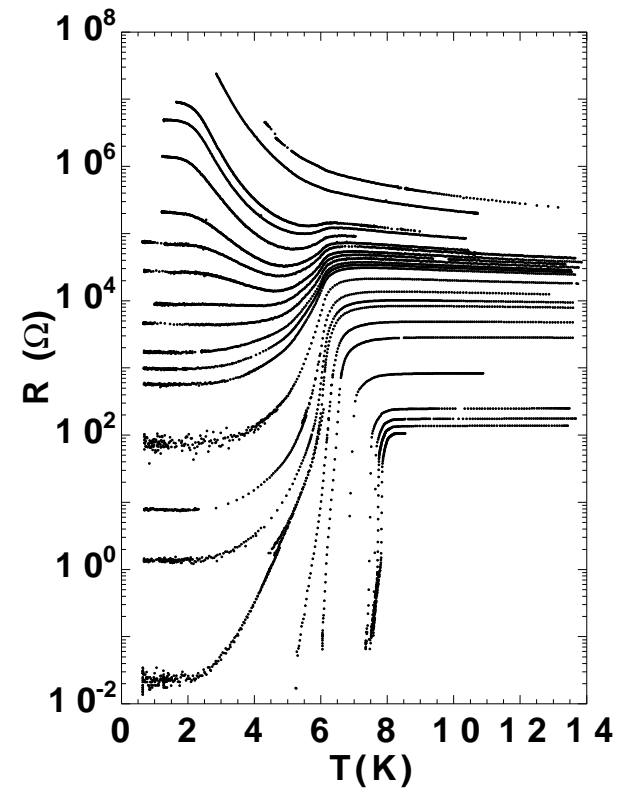

Figure 2: Reprinted from C. Christiansen, L. M. Hernandez, and A. M. Goldman, Phys. Rev. Lett., 88, 37004 (2002). Evolution of the temperature dependence of the resistance for a series of Ga films. Film thicknesses range from 12.75 A to 16.67A and increases from top to bottom. The leveling of the resistance once superconductivity is destroyed (zero resistance curves) is not consistent with conventional wisdom. Note that the plateau value of the resistivity increases as the distance from the superconducting phase increases. 


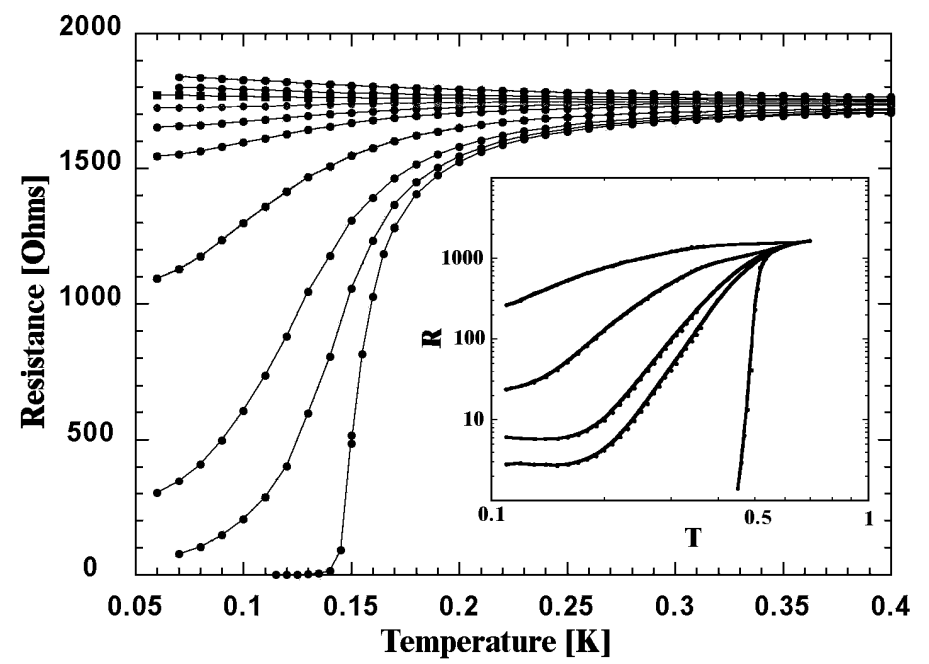

Figure 3: Electrical resistance of MoGe thin film plotted vs temperature at $\mathrm{B}=0,0.5,1.0,2.0$, 3.0, 4.0, 4.4, 4.5, 5.5, $6 \mathrm{kG}$. The sample becomes a superconductor at $0.15 \mathrm{~K}$ in zero field but for fields larger than about $4.4 \mathrm{kG}$ the sample becomes insulating. At fields lower than this but other than zero, the resistance saturates. The saturation behavior is better shown in the inset for another sample with a higher transition temperature. The inset shows data for $\mathrm{B}=0,1.5,2$, 4 ,and $7 \mathrm{kOe}$. At higher field, this sample is an insulator. Main figure reprinted from A. Yazdani and A. Kapitulnik, Phys. Rev. Lett. 74, 3037 (1995), while the inset is from, Phys. Rev. Lett. 76. 1529 (1996). 


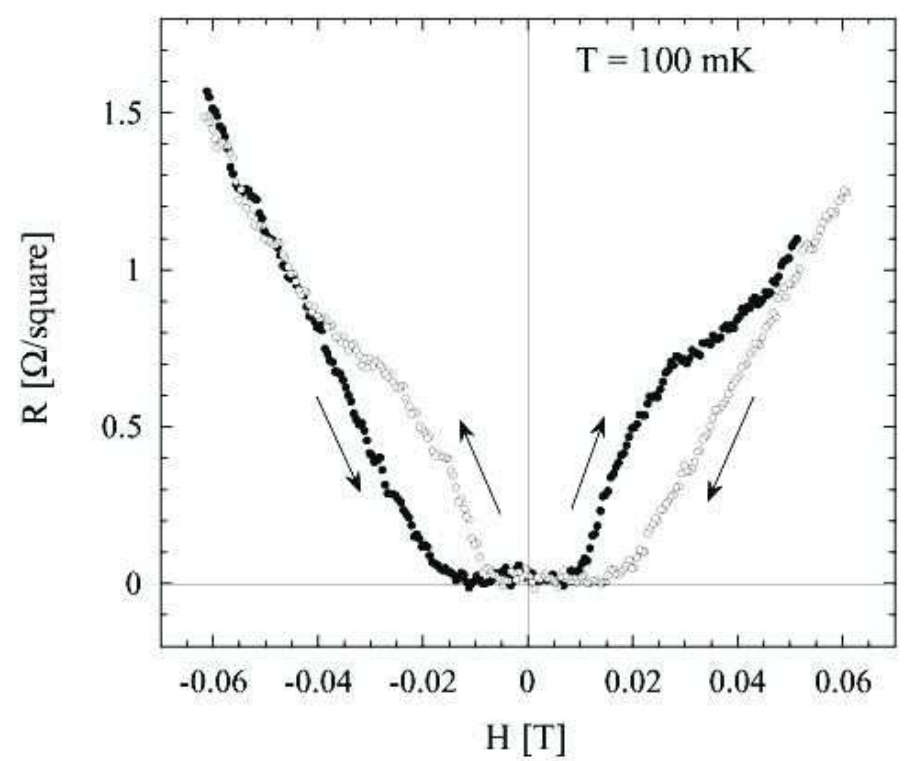

Figure 4: Reprinted from Phys. Rev. B, 64, 60504-1 (2001). Magnetoresistance at $T=80 \mathrm{mK}$ near the lower critical field, $H_{M}=0.018 T$ of a $40 \AA$ sample of $\mathrm{Mo}_{43} \mathrm{Ge}_{57}$ sandwiched between insulating layers of amorphous $\mathrm{Ge}$ on $\mathrm{SiN}$ substrates. Arrows indicate the direction of the field sweep. The curves are all shifted by 87 Oe to account for trapped flux in the $16 \mathrm{~T}$ magnet. The vanishing of the resistivity at $H_{M}=0.018 T$ is interpreted as the transition to the true superconducting state.
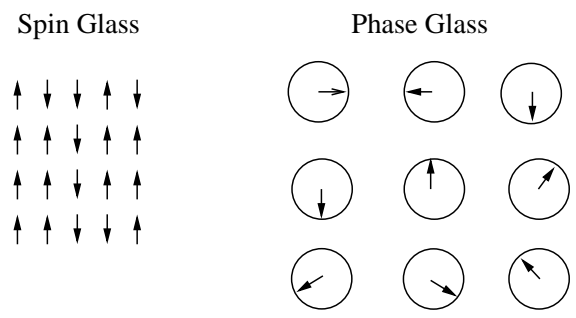

Figure 5: Patterns of spin and phase ordering in a spin glass and in a phase or rotor glass. In a spin glass, the spins point up or down but randomly such that the net magnetization vanishes. In the rotor glass it is the phases that point along random non-collinear directions in the $x-y$ plane, for example. 